\title{
Planning of spatially-oriented locomotion following focal brain damage in humans: A pilot study
}

\author{
Halim Hicheur ${ }^{\mathrm{a}, *}$, Carole Boujon ${ }^{\mathrm{a}}$, Cuebong Wong ${ }^{\mathrm{b}}$, Quang-Cuong Pham ${ }^{\mathrm{b}}$, \\ Jean-Marie Annoni ${ }^{\mathrm{c}}$, Titus Bihl ${ }^{\mathrm{d}}$ \\ a Unit of Sport and Movement Sciences, Dept. of Medicine, University of Fribourg, Switzerland \\ b School of Mechanical and Aerospace Engineering, Nanyang Technological University, Singapore \\ c Chair of Neurology, Dept. of Medicine, University of Fribourg \& HFR, Switzerland \\ ${ }^{\mathrm{d}}$ Neurorehabilitation Unit, Hôpital Fribourgeois (HFR), Switzerland
}

\section{H I G H L I G H T S}

- Motor impairments following brain damage are well documented.

- In particular, hemiparesis is a common feature of stroke patients. However, the navigational abilities (here, the ability to plan a path towards a distant target with vision or blindfolded) of such severely disabled patients have been poorly investigated.

- Here, we report that hemiparetic patients with significant lesions of the sensory-motor system following focal brain damage are not impaired during spatially-oriented locomotion performed with vision or blindfolded.

- This suggests that the motor cortex is involved in the sensorimotor implementation of locomotion but is not involved in the path planning stage in humans.

- Such findings open new avenues for post-stroke rehabilitation where specific tests (i.e., adapted versions of our paradigm) may allow parallel assessment of cognitive and locomotor functions recovery after stroke.

\begin{abstract}
Motor impairments in human gait following stroke or focal brain damage are well documented. Here, we investigated whether stroke and/or focal brain damage also affect the navigational component of spatially oriented locomotion. Ten healthy adult participants and ten adult brain-damaged patients had to walk towards distant targets from different starting positions (with vision or blindfolded). No instructions as to which the path to follow were provided to them. We observed very similar geometrical forms of paths across the two groups of participants and across visual conditions. This spatial stereotypy of whole-body displacements was observed following brain damage, even in the most severely impaired (hemiparetic) patients. This contrasted with much more variability at the temporal level. In particular, healthy participants and non-hemiparetic patients varied their walking speed according to curvature changes along the path. On the contrary, the walking speed profiles were not stereotypical and were not systematically constrained by path geometry in hemiparetic patients where it was associated with different stepping behaviors. These observations confirm the dissociation between cognitive and motor aspects of gait recovery poststroke. The impact of these findings on the understanding of the functional and anatomical organization of spatially-oriented locomotion and for rehabilitation purposes is discussed and contextualized in the light of recent advances in electrophysiological studies.
\end{abstract}

\footnotetext{
Abbreviations: VI, visual walking condition; BF, blindfolded walking condition; PH, hemiparetic patients; PN, nonhemiparetic patients; CO, control group (healthy participants); SN, step's number; SLR, step length ratio; SDR, stance duration ratio; ST, straight trajectories; LC, low curvature trajectories; MC, moderate curvature trajectories; $\mathrm{HC}$, high curvature trajectories; ATD, average trajectory deviation (mean of spatial variability around the mean trajectory across trials and subjects); MTD, maximal trajectory deviation (maximum of spatial variability around the mean trajectory across trials and subjects); ATS, average trajectory separation (mean distance between mean trajectories of two visual conditions or two groups of participants); MTS, maximal trajectory separation (maximal distance between mean trajectories of two visual conditions or two groups of participants); AMPVEL, amplitude of velocity variations during locomotor path completion.

* Corresponding author. Fax: +41 264268135

E-mail address: halim_hicheur@yahoo.fr (H. Hicheur).
} 


\section{Introduction}

How does the brain store spatial information about our position and orientation in our surrounding environment? How do we find or plan our way from one particular spatial position to another? Answers to these questions have come mainly from animal studies where different types of cells in the rat hippocampus and in neighboring regions (e.g., para-hippocampus and entorhinal cortex) were found to signal various spatial attributes [see Ref. [1] for a recent review]. These include the position of the animal in the room (the so-called "place cells"), its spatial orientation ("head direction cells") and, the metrics or the boundaries of the surrounding space ("grid" and "border" cells, respectively). Clinical and experimental data in humans do however suggest the role of a more distributed network during active navigation, as evidenced by the absence of behavioral deficits of hippocampal patients in path integration tasks [2,3] or by the role played by basal ganglia in the steering of blindfolded walking in circles [4]. In the case of path integration tasks, participants are required to keep track of a reference location using self-motion cues (for example by asking them to return to their starting position after having been passively/actively displaced along a path without vision). Importantly, the instructions provided to participants can affect the way navigational abilities are measured [5] and the navigation performance. For instance, the instruction to "maintain the path in mind" used in some path integration paradigms [3] during the learning phase may force participants to update their position with reference to the imagined path (a "map" representation of their body displacement in space, an allocentric strategy). In the absence of such (explicit) instructions, participants might spontaneously estimate their starting position by updating their position step by step with reference to the initial starting position (an egocentric or route strategy). As a consequence, assessing spatial navigation performance using path integration tasks is problematic because of possible interferences between these spatial processing/memory strategies, hence making it unclear whether, under natural conditions, participants would have memorized spatial attributes of the environment, spatial attributes of their displacements within the environment, or some combination thereof.

This problem can be overcome by asking participants to generate "spontaneous" walking behaviors. In previous years, we tested a simple goal-oriented task of walking toward distant targets (either doorways or arrows placed on the ground). Importantly, no specific constraint was imposed on healthy participants in terms of the path they were to follow. Participants had to perform these tasks using their vision or blindfolded [6,7]. Strikingly, we observed that the generated locomotor paths were similar across visual conditions (with vision or blindfolded) and that neither walking speed nor walking direction (forward or backward) significantly affected the shape of these paths [8]. The recorded body trajectories could be predicted by a combination of feed-forward and feed-back mechanisms, dedicated to accounting for the "global" (path-planning) and "on-line" contributions (visual guidance) to locomotor paths formation $[7,9]$. This suggests a dissociation between spatial cognition and sensorimotor control mechanisms at work during spatial navigation. Stereotyped locomotor trajectories were reported in adolescents and adults but not in children under 11 years [10], showing that path planning develops in late childhood (well after gait maturation), which suggests a distinct development of path planning vs gait motor stability abilities.

Understanding the potential interferences between cognitive and motor processes is of particular importance following stroke [11]. Here, we propose a proof of concept analysis dedicated to measuring the navigational performance of patients with motor and cognitive deficits following brain damage. Given the dissociation between motor and navigational components suggested by our previous findings, we were expecting that the shape of body trajectories in space, which mostly reflect spatial cognition processing (path planning), would remain unaffected by such motor deficits. In contrast, the motor implementation of these trajectories (e.g., the stepping behavior) would be affected by such deficits which, in our model, would result in greater variability around the mean trajectory.

\section{Materials and methods}

\subsection{Participants}

Ten patients (aged between 28 and 68 years, six females/four males) with chronic brain lesions following cerebrovascular events, and ten age/gender-matched healthy people (aged between 28 and 70 years, without any history of neurological disease) volunteered to participate in this experiment. All patients fulfilling the exclusion/inclusion criteria defined prior to experimental recordings were asked to participate in the study. The inclusion criteria were as follows: survivors of a first-time cerebrovascular event resulting in structural supratentorial or infratentorial cortical lesions (see Table 1 for detailed information about patients and Supplementary material for MRI templates), admitted to the in-patient rehabilitation unit of the Fribourg Cantonal Hospital (HFR), aged between 18 and 80 years, able to understand the meaning of the study and to follow instructions, and with good walking ability. Exclusion criteria were as follows: acute health problems which would interfere with the reliability of the task (infections, decompensated diabetes, etc.), questionable cardio-pulmonary status (cardiac failure, pulmonary embolism, oxygen therapy), patients with acute vigilance and spatial disorders (confusion, disorientation, etc.) at the time of the experimental recordings, patients with eye disorders and non-corrected vision problems (advanced macular degeneration, blindness, etc.), and pregnancy.

All selected patients exhibited paresis in the first week following brain damage. Five exhibited hemiparesis at the time of the experimental recordings which took place more than two months after the onset of stroke. They could walk at least $1000 \mathrm{~m}$ without the need to stop for rest. All participants had normal or corrected-to-normal vision. All patients exhibited residual cognitive deficits at the time of evaluation: four had aphasia, one had spatial neglect, two had memory impairment and six had dysexecutive syndrome. Longstanding attentional deficit was present in three patients. Nevertheless, all of them understood the information and orders given during the enrolment and test processes. Participants gave their informed consent prior to their 
Table 1

Demographic and neurological data of the 10 patients. 'Side' stands for the side of lesion: $\mathrm{L}=\mathrm{Left}, \mathrm{R}=\mathrm{Right}$. Delay means post stroke delay until gait evaluation and is given in months. ED = Executive dysfunction. Concerning site of lesion: $\mathrm{PFC}=$ prefrontal cortex; $\mathrm{M} 1=$ Primary motor Cortex, $\mathrm{PO}=\mathrm{Parieto}-\mathrm{occipital}$ cortex, $\mathrm{BG}=\mathrm{Basal}$ ganglia; $\mathrm{TO}=$ Temporo-occipital cortex; $\mathrm{PTO}=$ Parieto-temporo-occipital Cortex; FP = Fronto-parietal cortex; CRB = Cerebellum.

\begin{tabular}{|c|c|c|c|c|c|c|c|c|c|}
\hline \multicolumn{4}{|c|}{ Patients Gender Age Aetiology } & \multicolumn{2}{|c|}{ Delay Paresis } & \multicolumn{3}{|c|}{ FAC score Side Cognitive deficit } & \multirow{2}{*}{$\begin{array}{l}\text { Site of lesion } \\
\text { PFC, M1, insula, parietal cortex, BG }\end{array}$} \\
\hline P01 & $\mathrm{F}$ & 60 & Haemorrhagic stroke & 4 & No & 6 & $\mathrm{R}$ & Spatial neglect, attention, ED & \\
\hline P02 & $\mathrm{F}$ & 28 & Ischemic stroke & 8 & No & 6 & $\mathrm{R}$ & Attention, anterograde amnesia & TO, BG, CRB \\
\hline P03 & M & 66 & Ischemic stroke & 8 & Yes & 5 & $\mathrm{~L}$ & Fluent aphasia, apraxia, ED & M1, insula, BG \\
\hline P04 & M & 49 & Ischemic stroke & 5 & Yes & $4-5$ & $\mathrm{~L}$ & Non-fluent aphasia, apraxia, ED & M1, insula, PTO \\
\hline P05 & M & 67 & Meningioma surgery & 8 & No & 6 & $\mathrm{~L}$ & Attention & FP, involvement of M1 \\
\hline P06 & $\mathrm{F}$ & 57 & Subarachnoid haemorrhage & 8 & No & 5 & $\mathrm{R}$ & Motor aphasia, attention, anterograde amnesia, ED & FP \\
\hline P07 & M & 68 & Haemorrhagic stroke & 2 & Yes & 6 & $\mathrm{~L}$ & Mild learning deficit & M1/insula \\
\hline P08 & $\mathrm{F}$ & 60 & Vasculitis & 13 & Yes & 4 & $\mathrm{~L}$ & Anxiety, ED & BG \\
\hline P09 & $\mathrm{F}$ & 30 & Ischemic stroke & 11 & Yes & 6 & $\mathrm{~L}$ & Global aphasia, left hand apraxia, & M1, PO, BG \\
\hline P10 & $\mathrm{F}$ & 51 & Subarachnoid & 2.5 & No & 6 & $\mathrm{R}$ & ED & PFC, insula and left BG \\
\hline
\end{tabular}

inclusion in the study. Experiments conformed to the Code of Ethics of the Declaration of Helsinki and were approved by the Commission cantonale d'Ethique de la Recherche sur l'Etre humain (VD, Switzerland) and registered under reference NCT02263560 on the NIH ClinicalTrials.govClinicalTrials.gov database. Importantly, all patients could walk autonomously (one hemiparetic patient walked with a cane) as measured by a Functional Ambulation Categories (FAC) walking test [12], for which all patients had scores equal or superior to 4 (on the 6-point FAC scale). In order to test their ability to walk blindfolded, we asked patients to walk several steps whilst blindfolded in a room, to turn around and to walk back with and without physical assistance (all patients succeeded in this test). The patients were included in the study only when they expressed the feeling of safety (subjective) and when no nearfall event occurred (objective). All patients but one stayed at least one week at HFR, and returned home at least four weeks before the experimental recordings. P07 was tested few days before he returned home having spent two months at HFR. Importantly, all patients were followed by the same neurorehabilitation team.

\subsection{Protocol}

The experiments took place in a laboratory, the dimensions of which were $8.7 \times 6 \times 3.3 \mathrm{~m}$ (length, width and height respectively). The protocol was similar to the one used in our previous studies [see Refs. [6,7] and Supplementary material]. Briefly, participants had to start from one of three fixed positions in the laboratory (left, center or right) and to walk toward a distant target indicated by an arrow placed on the ground (see Fig. 1A). The dimensions of the arrow were $1.20 \times 0.25 \mathrm{~m}$ (length and width, respectively). The arrow was placed at a specific $(x, y)$ position in the room with a particular orientation (South, East, North and West, respectively $\mathrm{S}, \mathrm{E}, \mathrm{N}$ and $\mathrm{W}$ ). In the blindfolded condition, the participant first observed the arrow while standing at the starting position. This observation period typically lasted less than three seconds. When he (or she) was ready, he closed his eyes and attempted to complete the task without vision. The starting signal was given by the experimenter by touching the participant's shoulder with his hand (for both "visual" and "blindfolded" conditions).

\subsection{Experimental conditions}

Every participant generated 114 trajectories. For the "straight targets", participants had to perform 18 trials: one central starting position $(\mathrm{C}) \times$ three arrow positions $(1,2$ and 3$) \times$ one arrow orientation $(\mathrm{N}) \times$ two visual conditions (visual VI or blindfolded $\mathrm{BF}) \times$ three repetitions $=18$ trajectories. Concerning the "angled targets", they had to perform 96 trials: two starting positions (L and $\mathrm{R}$ ) $\times$ two arrow positions ( 2 and 3$) \times$ four arrow directions $(\mathrm{S}$, $\mathrm{E}, \mathrm{W}$ and $\mathrm{N}) \times$ two visual conditions $(\mathrm{VI}$ and $\mathrm{BF}) \times$ three repetitions. The trials were randomized (in terms of starting position, targets' position and orientation, visual condition and repetitions) in order to avoid any learning effect. These were recorded in two experimental sessions on different days (P04 took part in three experimental sessions). In addition to the inter-trial interval (which typically lasted 10-20 s), a rest period of five minutes occurred at the middle of the experimental session. A total of 2280 trajectories (1140 in patients and 1440 in controls) were recorded. Because of problems in data acquisition (with markers missing for at least one second), 22 trials (out of 2280) were excluded from the analysis; this involved six trials from healthy participants and 16 trials from patients.

\subsection{Experimental recordings}

Three-dimensional positions of light reflective markers were recorded at a $120 \mathrm{~Hz}$ sampling frequency using an optoelectronic Optitrack motion capture system (Natural Point Inc., Oregon, USA) wired to 15 cameras. Six markers were attached to motion capture suits or foot wraps (respectively) through velcro-friendly surfaces (Optitrack). Two were placed on the left and right shoulders at the level of the left and right acromions. They were used to study whole body trajectories in space [6,7]. Two markers were placed at the level of the heel and third toe of each foot. Participants wore a headset which prevented hearing sounds from outside.

\subsection{Data analysis}

Most of the following methods were presented in previous studies [6,7]. We will describe here the main methodological procedures which allowed us to analyze and compare trajectories produced in the different conditions and across healthy (control) participants ( $\mathrm{CO}$ ) and patients (hemiparetic $\mathrm{PH}$ and non-hemiparetic PN). The reader is referred to $[6,7]$ and to the Supplementary material for further details.

\subsection{General parameters and stepping behavior}

The length of the whole-body trajectories in space, the movement execution duration and the steps' parameters were computed. We used heel strike and toe off events to define steps [24]. These events were derived from the time course of heel and toe $Z$ position profiles and corresponded to the local minima of these two signals. We considered one step as the interval separating two successive heel strikes of the same foot and computed the feet positions at these particular events. The number, length and stance duration of left (non-paretic limb in PH patients) and right (paretic limb) steps were computed separately. The total number of steps (SN) and the Step Length/Stance Duration (non-paretic/paretic) Ratios (SLR and SDR, respectively) were computed to document the stepping behavior and the potential gait asymmetries (expected in PH patients in particular). 

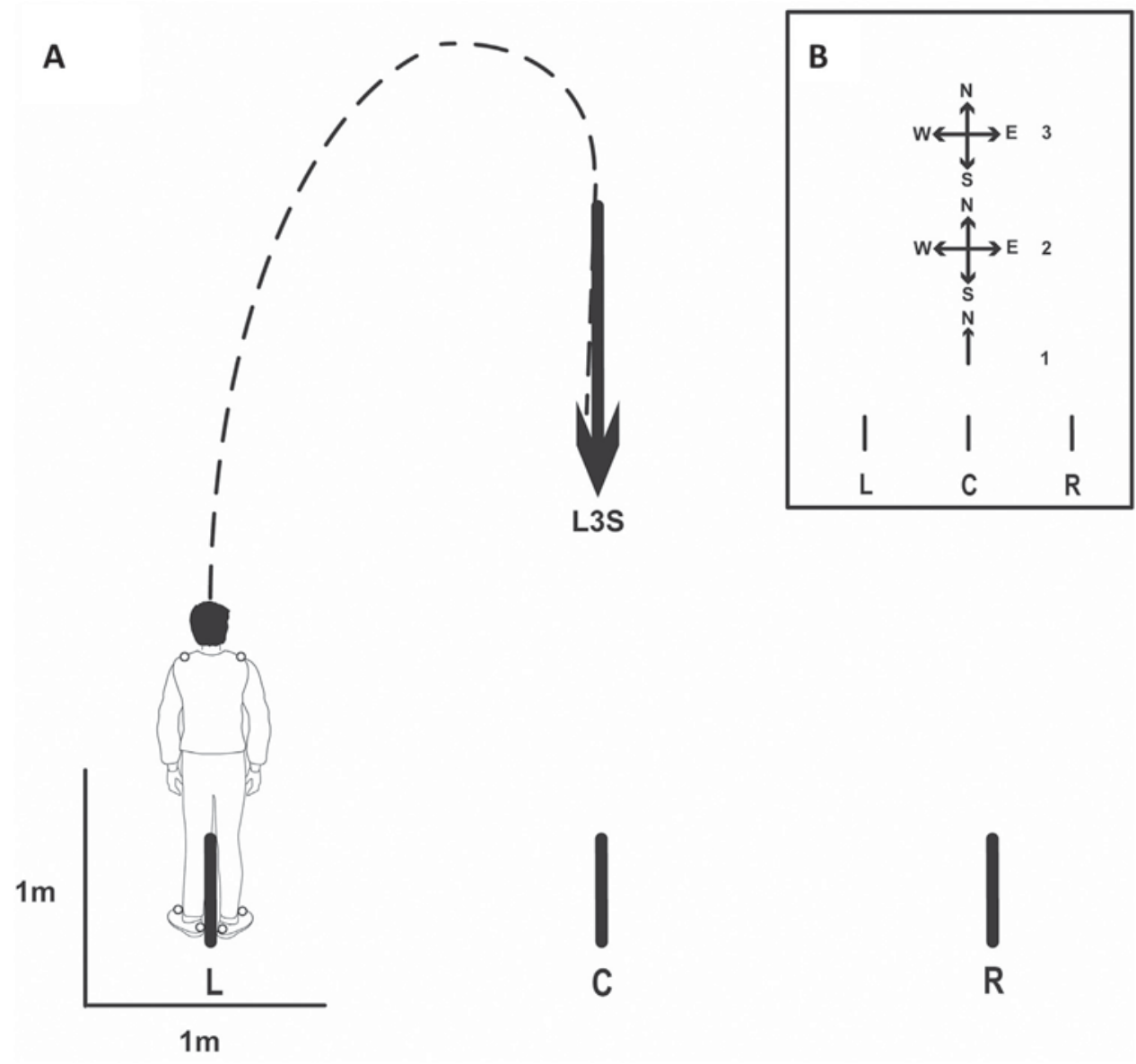

\subsection{Categorization and computation of the trajectories}

Here, the tested trajectories were classified according to the amount of body rotation they required. Four categories were distinguished: quasi-straight trajectories ST, low LC, moderate MC and highly HC curved trajectories. The beginning $(t=0)$ of each trajectory was set to the time instant when the participant crossed the $X$-axis at $y=0.5$ (the average length of the first "straight-ahead" step). In order to have the same criterion for the VI and BF conditions, the end of each trajectory $(t=1)$ was set to the time instant when the participant's speed became less than $0.06 \mathrm{~m} \mathrm{~s}^{-1}$ (this value was less than $5 \%$ of the average nominal walking speed). We chose this strictly positive threshold because of the small residual movements of the upper body occurring after participants stopped walking. When a derivative of the position was needed (to compute velocity profile, for instance), a second-order Butterworth filter with cut-off frequency $6.25 \mathrm{~Hz}$ was applied before the derivation.

\subsection{Spatial and temporal attributes of the locomotor trajectories}

The reader is referred to the supplementary material for details of the calculation of the following parameters. The spatial variability of the actual trajectory around the average trajectory (averaged across repetitions and subjects) was measured using the average and maximal trajectory deviation parameters (ATD and MTD), for every particular target. The comparison of the average trajectories recorded in two conditions (VI and $\mathrm{BF}$ ) or between patients and healthy participants in the same condition were measured using the average and maximal trajectory separation parameters (ATS and MTS). These parameters are expressed in centimeters. At the temporal level, the computation of the variability around the average velocity profiles focused on investigating whether participants varied their walking speed at similar instants/positions along the trajectory. This was quantified using the average and maximal velocity deviation parameters AVD and MVD (expressed in meters/s). The observation of quasi-constant walking velocities in hemiparetic patients obviously resulted in high AVD/MVD values (which measure deviations from average velocity profiles) across sub-groups of patients. Therefore, we also measured how much walking velocity varied during path completion. This was done by computing the amplitude of walking speed variations (AMPVEL) during path completion for every trial. Importantly here, high-frequency oscillations induced by stepping activity were removed to focus specifically on the global variation of the walking speed induced by curvature variations along the path $[13,14]$. This was done by individually adjusting the cut-off frequencies of the second-order Butterworth filter used when computing the velocity 
profiles (with a fixed value of $0.5 \mathrm{~Hz}$ for healthy participants and in the $0.2-0.5 \mathrm{~Hz}$ range for patients).

\subsection{Statistical tests}

All statistical comparisons were done using the Statistica 8.0 software package (Statsoft ${ }^{\circledR}$ ). We performed repeated measurement analyses of variance (ANOVA) to compare the parameters (ATD, MTD, AVD and MVD) calculated for the 2258 recorded trials (dependent variables: four categories $\times$ two visual conditions $\times$ three repetitions; categorical variables: two groups). These comparisons allowed us to quantify the effects of the magnitude of curvature (categories ST, LC, MC and HC), the visual condition (VI vs $\mathrm{BF}$ ) and the group (patients $\mathrm{PH}+\mathrm{PN}$ vs $\mathrm{CO}$ participants) on the spatial and temporal attributes of the trajectories. A second series of ANOVA analyses were performed to compare general (e.g., walking speed, travelled distances and walking duration when completing a path) and local step parameters (e.g., number of steps to complete a path, step length/duration ratios). Indeed, these parameters (except for the step length/duration ratios) were obviously more dependent upon initial distances (between the starting point and the target position/orientation) than on the magnitude of curvature. Thus, trials were categorized according to these distances into 11 categories of trajectories ( 11 categories $\times$ two visual conditions $\times$ three repetitions; categorical variables: two groups). Before performing each of these repeated-measures ANOVA comparisons on the patients' data, we first checked for the homogeneity of variances by performing a Mauchley's sphericity test. The normality of each of the computed distributions was then tested using Kolgomorov-Smirnov tests. If both hypotheses (variance and normality) were respected, we performed the ANOVA comparisons.

When statistically significant differences $(p<0.05)$ were observed between sub-groups of participants (e.g., $\mathrm{PH}$ and PN patients), the ANOVA comparisons were performed a second time on the population of patients only $(N=10)$, to test for the effect of hemiparesis on the computed variables. Here, the tests of sphericity and normality were performed on the patients' data. Any atypical behavior observed for a particular patient or healthy participant (detected or not by these comparisons) will be mentioned in the text.

\section{Results}

\subsection{General parameters and stepping behavior}

On average, hemiparetic patients walk more slowly than healthy controls and non hemiparetic brain lesion patients [15]. We observed the same group effect here: the mean walking speed of CO was equal to $0.98 \pm 0.11$ and $0.85 \pm 0.12 \mathrm{~m} / \mathrm{s}$ (visual and blindfolded trials, respectively) while it was equal to $0.88 \pm 0.15$ and $0.74 \pm 0.15 \mathrm{~m} / \mathrm{s}$ in $\mathrm{PN}$ and to $0.59 \pm 0.19$ and $0.47 \pm 0.17 \mathrm{~m} / \mathrm{s}$ in $\mathrm{PH}$ $(F(1,16)=14,879, p<0.01)$. We also observed a significant effect of the category of trajectories $(F(10,160)=58,394, p<0.01)$ on the walking speed (see Fig. 6A of the Supplementary material for mean values for all groups and categories), with the highest speeds being reached for the second and third straight targets (ST) and the lowest speeds being reached for the four most angled (HC) targets. The visual condition also significantly affected the walking speed $(F(1,16)=121,8, p<0.01)$. The (category $\times$ vision $)$ interaction effect was significant $(F(10,160)=8,0425, p<0.01)$ while the (category $\times$ group) interaction effect was not significant $(p>0.05)$. We did not observe any other interaction effect. The effect of hemiparesis on the walking speed was assessed by performing ANOVA on the patients' group. This effect was significant $(F(1,8)=6,87, p=0.03)$ : $\mathrm{PH}$ walked at a significantly lower speed than PN. We observed here a significant (category $\times$ hemiparesis) interaction effect $(F(10$, $80)=2149, p=, 029$ ) with walking speeds being nearly constant in the PH sub-group across the 11 tested categories of trajectories. However, the individual analysis of the walking speeds within each sub-group of patients revealed that PH patients P07 and P09 had walking speeds comparable to that of PN. This effect of hemiparesis can thus be attributed to PH patients P03, P04 and P08. Thus, hemiparesis significantly reduced the walking speed (compared to PN) which was constant across straight and angled targets for these three $\mathrm{PH}$ patients only.

These changes observed at the level of the walking speeds were associated with corresponding changes at the level of traveled distances and duration times. The detailed results of this analysis as well as those of the stepping behavior are described in the supplementary material. Briefly, we observed that the traveled distances were comparable across groups of participants. Thus, the lower average walking speed resulted in longer movement durations in PH patients.

The analysis of the stepping behavior provided observations comparable to that of other studies on hemiparetic gait [16]. The strongest marker of gait asymmetry in $\mathrm{PH}$ was a longer stance phase duration of the non-paretic limb. Importantly, we did not observe any statistically significant effect of the turning direction (with $\mathrm{PH}$ turning first with their paretic or non-paretic limbs for right and left turns, respectively) on all computed parameters, as reported in a recent study [16]. Taken together, these results revealed that, to generate trajectories of comparable distances, different stepping patterns were implemented in different groups of participants.

\subsection{Spatial attributes of the whole-body trajectories}

Typical trajectories observed for healthy participants and patients are depicted for four targets in Fig. 2 . The similar geometrical form of the whole-body trajectories across visual conditions and groups is remarkable. The absence of effect of cortical and subcortical lesions (see Fig. 2B5 and C5) on the geometrical form of trajectories both during VI and BF trials should be noticed, even for the hemiparetic patient P04 who suffers from important cortical lesions in the left hemisphere. In this patient and in the other $\mathrm{PH}$ patients, one could observe local oscillations during the trajectory which are due to the particular stepping activity of $\mathrm{PH}$ patients (see above). The similarity between healthy participants and patients' average trajectories on one hand, and between VI and $\mathrm{BF}$ average trajectories on the other hand, was quantified using the ATS and MTS parameters (Fig. 3A and B). The spatial trajectory separation between groups ranged between 4/5 (ATS/MTS) and $9 / 12 \mathrm{~cm}$ for the VI trials and between $3 / 6$ and $22 / 32 \mathrm{~cm}$ for the BF trials, respectively. The ATS and MTS parameters computed between visual conditions were of comparable magnitude. The spatial variability around the average trajectory was quantified using the ATD and MTD parameters (Fig. $3 \mathrm{~A}$ and B). These never exceeded $25 \mathrm{~cm}$ (ATD), $47 \mathrm{~cm}$ (MTD), $48 \mathrm{~cm}$ (ATD) and $84 \mathrm{~cm}$ (MTD) centimeters for VI and BF trials, respectively. The ANOVA comparisons revealed no effect of the group $(p>0.05)$. They revealed a significant effect of the category $(F(3,48)=47,23, p<0.01$ and $F(3$, $48)=34,53, p<0.01)$ and of the visual condition $(F(1,16)=110,3$, $p<0.01$ and $F(1,16)=142,8, p<0.01$ ) as well as an interaction (category $\times$ visual condition) effect $(F(3,48)=5,68, p<0.01$ and $F(3$, $48)=4,18, p=0.010$ ) on the ATD/MTD parameters, respectively. The variability increased with increasing curvature and for BF trials [as reported in previous studies, see Refs. [6,7]].

Taken together, these results indicate that all groups of participants generated similar forms of locomotor paths across categories and visual conditions. 


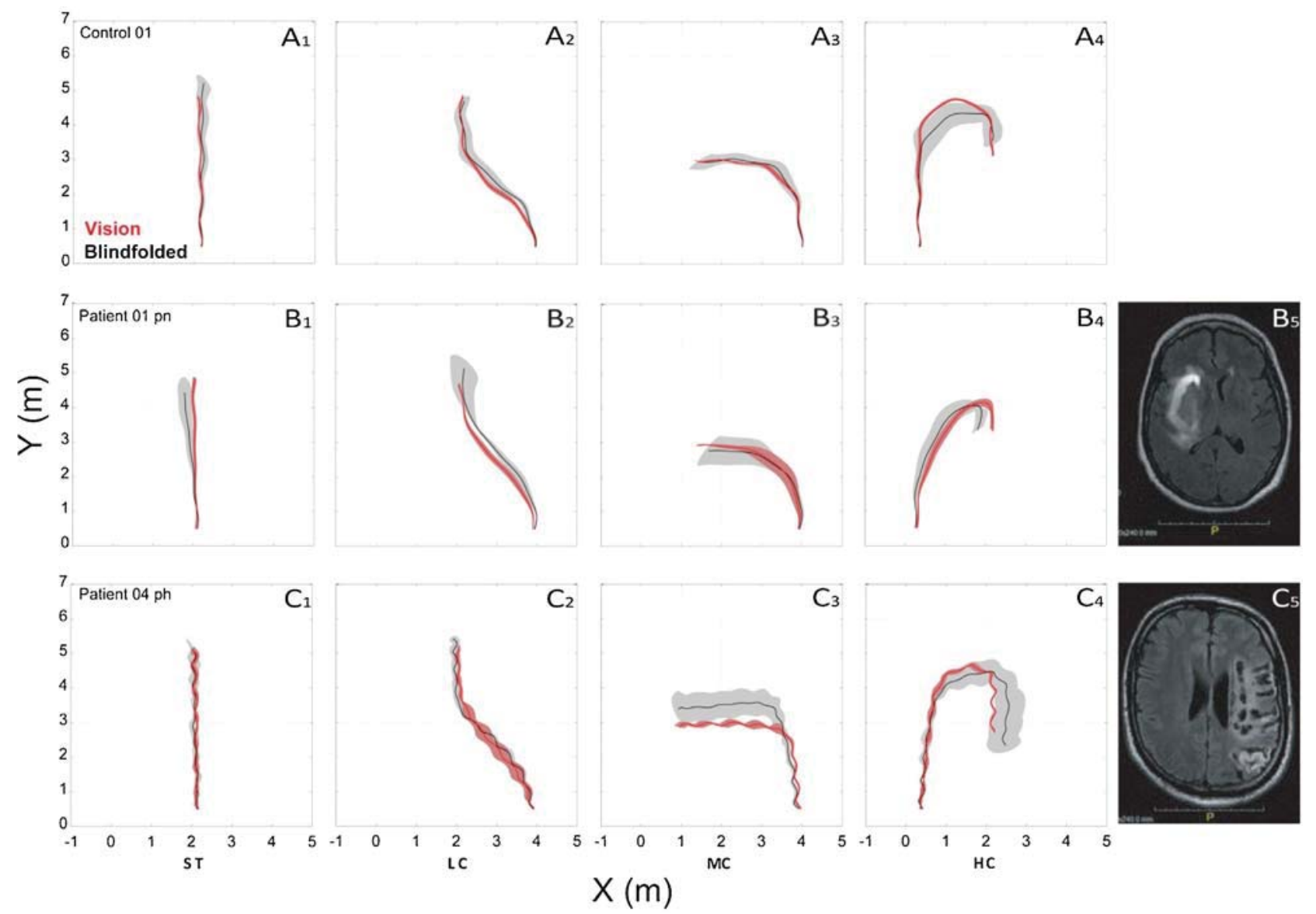

Fig. 2. Typical trajectories generated by control participants (A), non-hemiparetic (B) and hemiparetic (C) patients. Panels 1-4 indicate straight-ahead walking (ST), low curvature (LC), medium curvature (MC) and high curvature (HC) trajectories. Panel 5 indicate the lesioned brain area in patients (see Table 1 for details). Average trajectories performed for visual and blindfolded trials are represented by thick lines (red and black colors, respectively). The variability around the average trajectory is represented by the shadow region. High-frequency oscillations around the trajectory were observed in PH patients only and are associated with the specific stepping pattern of PH patients (see Supplementary material for details of gait pattern changes in all groups). Note the great similarity of the locomotor trajectories for all groups and visual conditions. Note also that the variability around these average trajectories (shadow region) is higher without vision for all groups and visual conditions.

\subsection{Temporal attributes of the whole-body trajectories}

The walking velocity variations during trajectory generation are depicted for the same participants and targets as those presented in Fig. 2 (Fig. 3). The first and main observation is the different velocity profiles of $\mathrm{PH}$ patients compared to $\mathrm{PN}$ and healthy participants. Indeed, not only do PH patients walk more slowly than the other groups, but they also maintain a constant velocity during their displacement in the room, for both "straight" and "angled" targets. This contrasts with similar velocity profiles between PN patients and healthy participants. The similarity between the velocity profiles across groups, categories and visual conditions has been quantified using the AVD and MVD parameters, respectively, (see Fig. 4C and D). Comparable values were observed between $\mathrm{CO}$ and $\mathrm{PN}$ groups while higher values were observed for PH. However, the ANOVA could not reasonably be performed for the whole dataset (AVD and MVD) as the Mauchley test for sphericity was positive (the variances of the patients' group were not homogeneous across groups). We therefore performed the ANOVA separately for the CO and the patients' groups. The only statistically significant effect for the $\mathrm{CO}$ was the one of the category of targets $(F(3,27)=16,76, p<0.01)$ on the MVD parameter (which was significantly higher for HC compared to ST category). The test for sphericity was positive for the patients' group for both AVD and MVD parameters. This can be explained by larger AVD/MVD variability within the PH subgroup. It can also be explained by a lack of sensitivity of the AVD/MVD parameters (which were dedicated to measure deviations from the mean velocity profile across individuals) in detecting different patterns of velocity profiles along individual path completion.

That is why we measured how much walking velocity varied during path completion by computing the amplitude of walking speed variations (AMPVEL, Fig. 4D). The test for sphericity was positive when applied to the whole AMPVEL dataset. We thus performed the ANOVA comparisons separately for $\mathrm{CO}$ and patients. The velocity variations ranged between 0.4 to $0.7 \mathrm{~m} / \mathrm{s}$ across categories and conditions for the $\mathrm{CO}$ group. We observed a significant effect of the category $(F(3,27)=33,42, p<0.01)$, of the visual condition $(F(1,9)=19,51, p<0.01)$ as well as an interaction (category $\times$ visual condition) effect $(F(3,27)=10,85, p<0.01)$ on AMPVEL. The range of velocity variations was comparable between $\mathrm{CO}$ and PN groups (Fig. 4D). We thus grouped together PN data with the (age-gender matched) CO data. The test for sphericity was negative. The ANOVA comparisons revealed no significant effect of the group $(p>0.05)$ and a significant effect of the category $(F(3,24)=53,67, p<0.01)$, of the visual condition $(F(1,8)=25,49, p<0.01)$ as well as an interaction (category $\times$ visual condition) effect $(F(3,24)=8,99, p<0.01$ ) on AMPVEL. Thus, both CO and PN varied significantly more their walking speed along the path for the highly curved trajectories (HC) and in VF trials. The test for sphericity performed on patients' data was positive, revealing that AMPVEL was not homogeneous in the patients' population. We observed that variability was particularly important in $\mathrm{PH}$ in BF trials and for the HC category (Fig. 4D). 

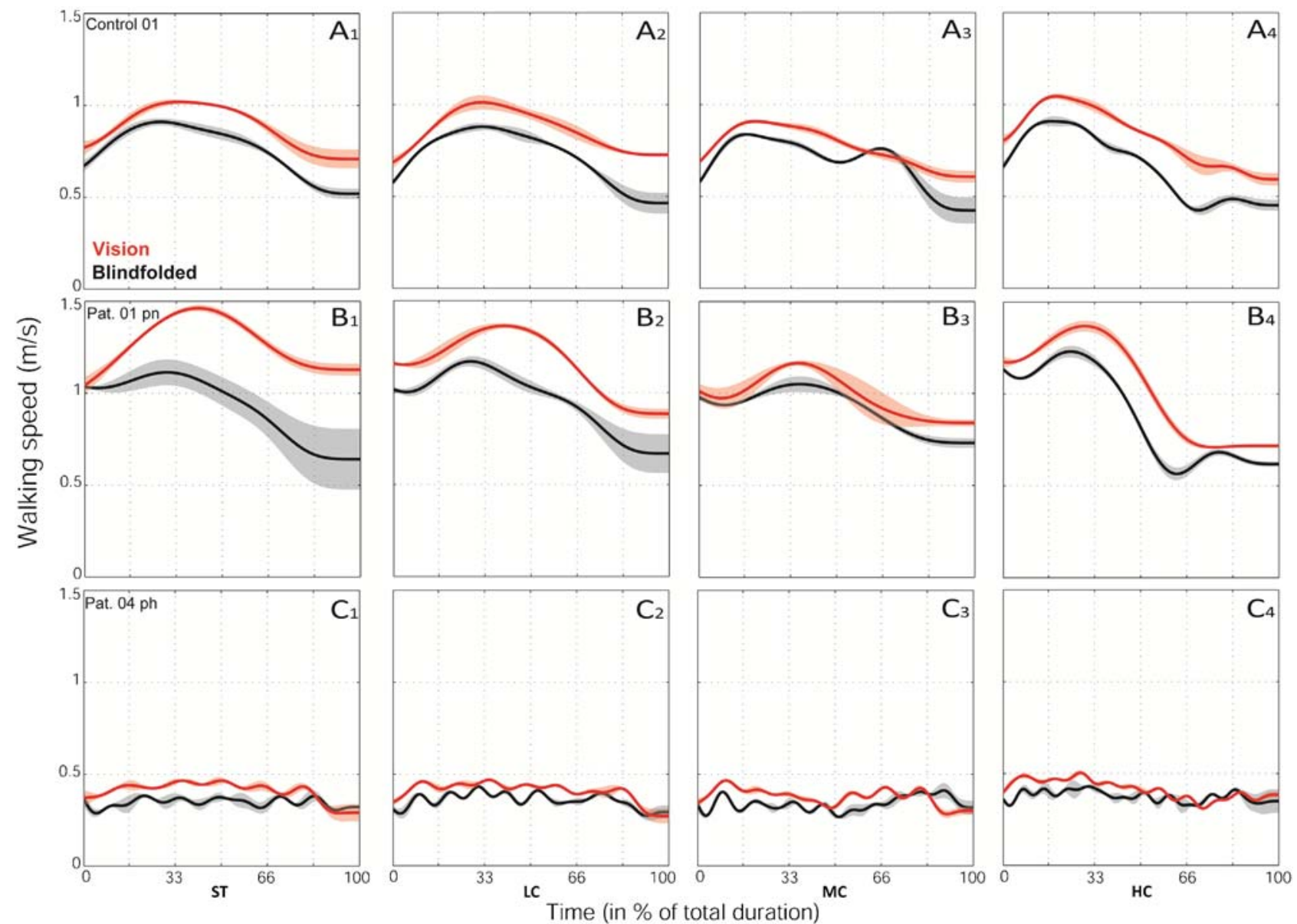

Fig. 3. Typical velocity profiles generated by control participants (A), non-hemiparetic (B) and hemiparetic (C) patients during trajectory completion. Same color code as Fig. 4. Note the quasi-constant walking velocity generated by the PH patient in performing the task, in contrast with the PN patients and the control group. This type of constant-velocity patterns was observed in patients P03, P04 and P08.

We thus repeated the sphericity test for the ST, LC and MC categories on one hand and for the HC category on the other hand, and both tests were negative on patients' data. The ANOVA comparisons performed for the first three categories revealed that AMPVEL was significantly smaller for PH compared to PN $(F(1,8)=6,32$, $p=0.036)$. We also observed a significant effect of the category $(F(2$, $16)=3,83, p=0.044)$, of the visual condition $(F(1,8)=23,56, p<0.01)$ as well as an interaction (category $\times$ visual condition) effect $(F(2$, $16)=3,6443, p=0.04964$ ) on AMPVEL. Velocity varied less during path completion for ST targets and in VF trials. The ANOVA comparisons performed for the $\mathrm{HC}$ target revealed a significant effect of the visual condition $(F(1,8)=33,77, p<0.01)$ on AMPVEL and a weak (but not significant) effect of the group $(F(1,8)=4,93, p=0.057)$. Thus, $\mathrm{PH}$ patients had larger intra-group variability than $\mathrm{CO}$ and $\mathrm{PN}$ patients (see also Supplementary material for similar observations reported at the level of the stepping behavior). Overall, variations in walking velocity during path completion are significantly smaller in PH patients compared to PN and CO (Fig. 4D).

Taken together, these results show that, on average, $\mathrm{CO}$ and PN groups generate similar velocity patterns, while PH group generate different (and more variable) velocity patterns and vary their walking speed significantly less along the path (with patients P03-P04-P08 walking at quasi-constant velocities whatever the curvature variations along the path).

\section{Discussion}

Motor impairments in gaits of individuals following stroke or brain damage are well documented [see Ref. [15] for a recent review]. Here, we investigated whether these impairments also affect the cognitive (navigational) component of a spatially oriented locomotor task. Importantly, participants were free to choose any path allowing them to reach a distant (visible or memorized) target (with vision or blindfolded). As previously observed in healthy adult participants $[6-8,10]$, we observed very similar geometrical forms of paths across target positions and visual conditions. Remarkably, this spatial stereotypy of the locomotor trajectories was observed following brain damage, even in the most severely impaired (hemiparetic $\mathrm{PH}$ ) patients. This contrasted with much more variability at the temporal level. In particular, healthy participants and non-hemiparetic patients varied their walking speed according to curvature changes along the path. On the contrary, the walking speed profiles were not stereotypical and were not systematically constrained by path geometry in hemiparetic patients (walking velocity was almost constant in three PH patients) where it was associated with different stepping behaviors. This extension of our previous observations to patients with significant lesions of the sensory-motor system, and independently of the presence of non-navigational cognitive deficits, yields direct 

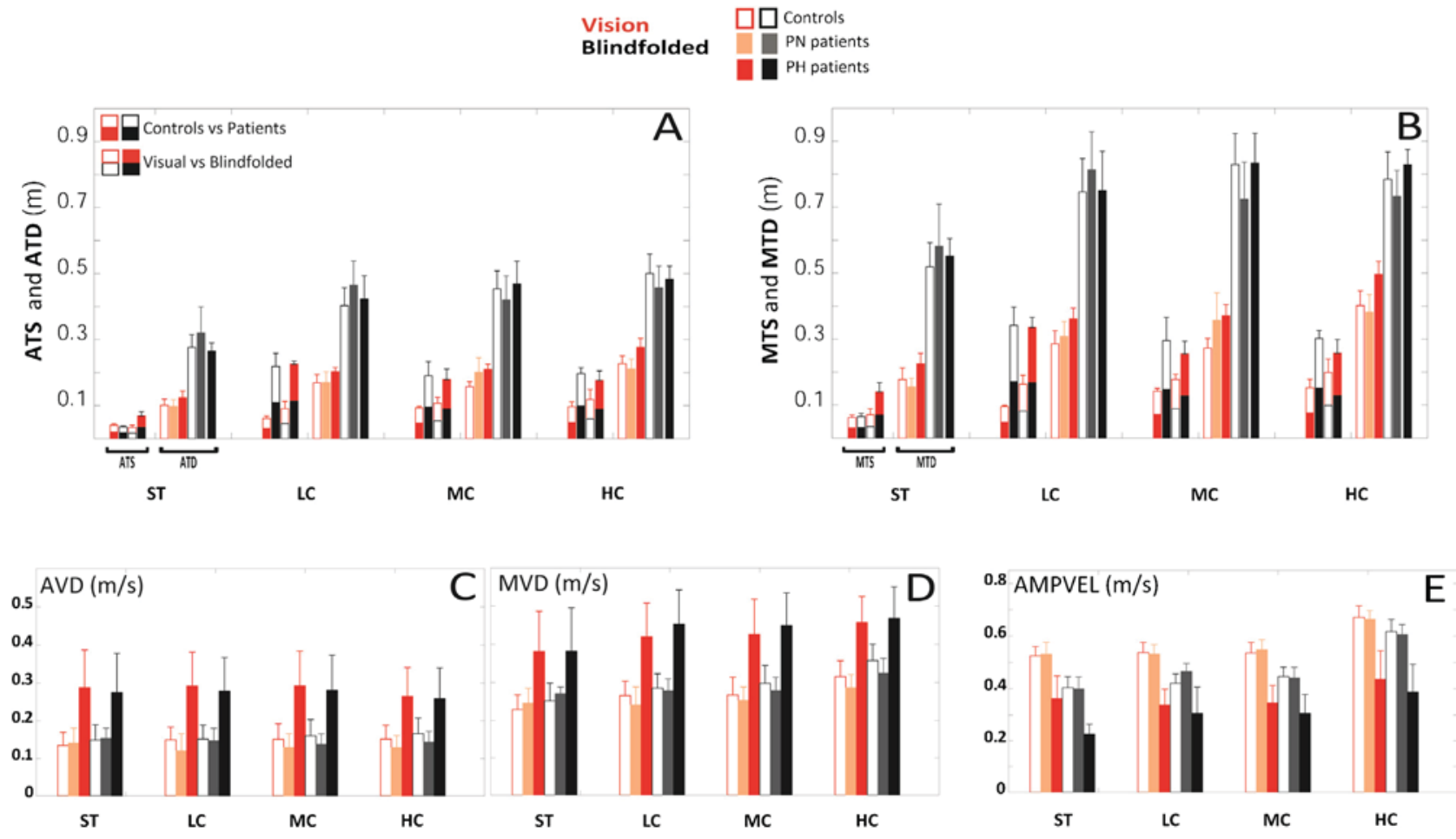

Fig. 4. Measures of the spatial (A-B) and temporal (C-E) variability of the walking trajectories across groups and visual conditions. ST, LC, MC and HC indicate straight-ahead walking, low curvature, medium curvature and high curvature trajectories, respectively. (A-B) Average and maximal distances between trajectories across groups or visual conditions (ATS and MTS, respectively: see insert in panel A for the code color used to display the Controls vs Patients and Visual vs Blindfolded comparisons, respectively). Average and maximal distances between trajectories across repetitions of all participants (ATD and MTD, code color similar to Fig. 3). Note that trajectory separation indices across groups or visual conditions never exceed, on average, $0.2 \mathrm{~m}$ (ATS) and that all patients do not differ from control participants' values. (C-D) Similar measurements performed for the velocity profile: note the systematically higher variability for PH patients only. E-Amplitude of velocity variations during path completion. Note that velocity varies significantly less in blindfolded trials and for $\mathrm{PH}$ patients across categories and visual conditions.

experimental evidence in humans that the motor cortex is involved in the sensorimotor implementation of locomotion but is not involved in the path planning/navigational stage. This has several implications that are discussed below.

\subsection{Navigational abilities following stroke or brain damage}

Van der Ham et al. [17] observed that $29 \%$ of post-mild stroke patients included in their study complained of spatial navigation impairments which could not be detected using most of the neuropsychological tests in common use. Such frequent complaint of stroke patients is related to the disorientation problems they experience when navigating in a familiar space in partial or complete absence of vision (like walking from their bed to the kitchen in the night). Importantly, this can occur even after full motor recovery, as recently reported by Han et al. [18] who described the case of a 72year old patient who experienced a sudden inability to navigate to the restroom, kitchen, or any familiar place after suffering a stroke. Notably, this patient with multiple acute ischemic brain lesions in the parietal and occipital lobes was blind for 30 years and did not exhibit any disorientation problems prior to the stroke. The disorientation problems were resolved within three days of treatment. The absence of persistent navigational problems even in hippocampal patients performing active locomotor or pointing tasks [2,3] can be related to the difficulty in assessing spatial memory and/or navigation abilities [5]. The goal-oriented task we tested here belongs to the latter type of abilities in the sense that all participants had both to plan and execute a whole-body displacement in a new environment. We did not observe differences between patients and healthy participants at the spatial (cognitive) level despite important differences at the execution (motor) level. In other words, we could not find any "path-planning" impairment even in patients having experienced strokes only two months before inclusion in the study. In a review paper, Krakauer [19] distinguished the post-stroke kinematical and dynamical potential troubles for arm movements and argued that the abnormal (hemiparetic) arm movements may suggest a deficit in transforming a planned trajectory into the appropriate joint angles. This distinction seems also to hold true for hemiparetic whole-body movements. However, we cannot exclude the possibility that patients with specific (e.g., hippocampal) lesions or patients at earlier time points after strokes would also exhibit path planning deficits. This should be tested in future studies. Besides, it could be argued that the constant walking speeds observed in three hemiparetic patients reveal planning deficits at the temporal level. While we cannot exclude this possibility, our results show a clear dissociation between spatial and temporal components of whole-body motion planning. This is further discussed below.

\subsection{Sensorimotor implementation of locomotor trajectories}

Another functional implication of the present study is related to the nature of the mechanisms underlying locomotor trajectory formation and control. We previously reported that in healthy participants the spatial stereotypy of locomotor trajectories does not rely on the availability of visual inputs $[7,9]$. Our present study extends these observations to patients and to healthy elderly people. Nevertheless, in previous studies we observed that vision is involved in minimizing the variations around the average (stereotyped) trajectories. We could predict both average trajectories and variability profiles around these trajectories using a model combining two modules accounting for the "global" (path-planning) and 
"on-line" contributions (visual guidance) to locomotor paths formation. Importantly, both modules rely on optimality principles already described for hand movements planning and control [20].

Other approaches do not assume such computational modules and propose a direct use of optic flow (alone or in combination with other visual variables) to guide locomotion and also allow for the prediction of a large range of locomotor trajectories [21]. The spatial stereotypy of trajectories observed during blindfolded locomotion questions the relevance of such approaches. It could be argued that the stereotyped behavior observed during blindfolded locomotion is the by-product of some preserved visual motor feedback loops. In any case, this would require translating the memorized (static) position/orientation of the locomotor goal into appropriate (dynamic) visuo-motor patterns, e.g., some non-visual computational processing. Adapting our paradigm and testing congenitally blind people might help to disambiguate the possibility of preserved visual-motor feedback loops. Besides, the observation of spatial stereotypy in hemiparetic patients does not support the hypothesis of preserved visual-motor feedback loops as these patients suffer from significant impairments of their locomotor patterns; rather, it seems that some higher order (cognitive) mechanism explains the spatial stereotypy of locomotor trajectories while visuomotor control loops would likely be involved in the on-line control of the steering behavior. Thus, the constant walking speeds observed in hemiparetic patients would represent an adapted visuomotor steering strategy rather than a path-planning deficit. At the modeling level, previous studies have suggested that, in both hand movements and locomotion, the velocity profiles along a predefined path are constrained by geometric quantities or principles (curvature or affine invariance) or optimization principles $[14,20,22]$. Within this context, the observation of similar geometrical forms of paths, but different velocity profiles, in hemiparetic patients further supports the existence of a dissociation between the spatial (path-planning) and temporal (sensorimotor implementation level) components of spatially-oriented locomotion in humans.

\subsection{Brain areas involved in the spatial and temporal aspects of spatially-oriented walking}

In a recent review paper mainly based on animal studies, Drew and Marigold [23] proposed an anatomical distinction of brain areas involved in different aspects of locomotion. Namely, they provided neurophysiological evidence for a different contribution of posterior parietal and motor cortices in the planning and execution of locomotion, respectively. In the cited studies, the planning level was mainly related to limb trajectory planning during obstacle avoidance tasks in cats (with vision). Our behavioral observations show that, in our patients, important brain lesions in the premotor and motor cortices do not affect path-planning mechanisms; this supports the propositions of Drew and Marigold [23] and goes even further as we suggest similar distinctions between planning (spatial) and execution (temporal) levels of locomotion but at the level of the whole-body trajectory. Besides, we observed that patients with focal lesions of the cerebellum or basal ganglia did not exhibit navigational deficits. Importantly, this absence of behavioral deficits at the planning level was observed even in the absence of vision. It should also be noted that more distributed cortical network is involved in the planning and execution of whole-body displacement. Taken together, these observations suggest that medio-temporal areas known to be involved in spatial processing (including the hippocampus, see Section 1) may play a critical role in providing a continuous "route to follow" signal to the motor cortex which would then translate this into motor commands. Following stroke, plasticity in the motor and somatensory cortices would result in a different sensorimotor implementation of the locomotor path. Whether "the path planning" stage is encoded in allocentric or egocentric coordinates cannot be answered using the present data. Future studies manipulating these different types of spatial representations may provide a deeper understanding of the functional and anatomical organization of spatially-oriented locomotion. Another limitation of our study is the relatively small-scale environment (comparable to daily locomotor tasks at home) in which participants performed the tasks.

\subsection{Implications for rehabilitation}

The potential dissociation between cognitive and motor aspects of gait recovery post-stroke must be further studied at different time points after stroke and for complex locomotor tasks. The findings of this pilot study are reminiscent of those reported by Belmonti et al. [10]: cognitive and motor components of human locomotion seem to evolve independently during a lifetime, with a stabilization of gait occurring earlier (around 4-5 years of age) than path planning (around 11-13 years of age in their study). When applied to focal neurological diseases affecting gait, this emphasizes the need to develop tests (i.e., adapted versions of our paradigm) allowing parallel assessment of cognitive and motor functions after stroke; this may help rehabilitation teams to better focus on the specific deficits of patients.

\section{Acknowledgments}

The authors would like to thank Dr Alan Chauvin for his help with the statistical analysis and two anonymous reviewers for their constructive suggestions and comments.

\section{Appendix A. Supplementary data}

Supplementary data associated with this article can be found, in the online version, at http://dx.doi.org/10.1016/j.bbr.2015.12.014.

\section{References}

[1] G. Buzsaki, E.I. Moser, Memory, navigation and theta rhythm in the hippocampal-entorhinal system, Nat. Neurosci. 16 (2013) 130-138.

[2] S. Kim, M. Sapiurka, R.E. Clark, L.R. Squire, Contrasting effects on path integration after hippocampal damage in humans and rats, Proc. Natl. Acad. Sci. U. S. A. 110 (2013) 4732-4737.

[3] Y. Shrager, C.B. Kirwan, L.R. Squire, Neural basis of the cognitive map: path integration does not require hippocampus or entorhinal cortex, Proc. Natl. Acad. Sci. U. S. A. 105 (2008) 12034-12038.

[4] C. Paquette, E. Franzen, G.M. Jones, F.B. Horak, Walking in circles: navigation deficits from Parkinson's disease but not from cerebellar ataxia, Neuroscience 190 (2011) 177-183.

[5] T. Wolbers, M. Hegarty, What determines our navigational abilities? Trends Cogn. Sci. 14 (2010) 138-146.

[6] H. Hicheur, Q.C. Pham, G. Arechavaleta, J.P. Laumond, A. Berthoz, The formation of trajectories during goal-oriented locomotion in humans. I. A stereotyped behaviour, Eur. J. Neurosci. 26 (2007) 2376-2390.

[7] Q.C. Pham, H. Hicheur, On the open-loop and feedback processes that underlie the formation of trajectories during visual and nonvisual locomotion in humans, J. Neurophysiol. 102 (2009) 2800-2815.

[8] Q.C. Pham, A. Berthoz, H. Hicheur, Invariance of locomotor trajectories across visual and gait direction conditions, Exp. Brain Res. 210 (2011) 207-215.

[9] Q.C. Pham, H. Hicheur, G. Arechavaleta, J.P. Laumond, A. Berthoz, The formation of trajectories during goal-oriented locomotion in humans. II. A maximum smoothness model, Eur. J. Neurosci. 26 (2007) 2391-2403.

[10] V. Belmonti, G. Cioni, A. Berthoz, Development of anticipatory orienting strategies and trajectory formation in goal-oriented locomotion, Exp. Brain Res. 227 (2013) 131-147.

[11] C. Chen, D. Leys, A. Esquenazi, The interaction between neuropsychological and motor deficits in patients after stroke, Neurology 80 (2013) S27-S34.

[12] M.K. Holden, K.M. Gill, M.R. Magliozzi, J. Nathan, L. Piehl-Baker, Clinical gait assessment in the neurologically impaired. Reliability and meaningfulness, Phys. Ther. 64 (1984) 35-40.

[13] H. Hicheur, A. Berthoz, How do humans turn ? Head body movements for the steering of locomotion, Proceedings of the IEEE-Robotics and Automatic Society International Conference on Humanoid Robots (2005) 265-270. 
[14] H. Hicheur, S. Vieilledent, M.J.E. Richardson, T. Flash, A. Berthoz, Velocity and curvature in human locomotion along complex curved paths: a comparison with hand movements, Exp. Brain Res. 162 (2) (2005) 145-154.

[15] S. Lauziere, M. Betschart, R. Aissaoui, S. Nadeau, Understanding spatial and temporal gait asymmetries in individuals post stroke, Int. J. Phys. Med. Rehabil. 2 (2014).

[16] M. Godi, A. Nardone, M. Schieppati, Curved walking in hemiparetic patients, J. Rehabil. Med. 42 (2010) 858-865.

[17] I.J. Van der Ham, N. Kant, A. Postma, J.M. Visser-Meily, Is navigation ability a problem in mild stroke patients? Insights from self-reported navigation measures, J. Rehabil. Med. 45 (2013) 429-433.

[18] Y.H. Han, M.C. Pai, C.T. Hong, Topographical disorientation in a patient with late-onset blindness with multiple acute ischemic brain lesions, J. Clin. Neurosci. 18 (2011) 283-285.
[19] J.W. Krakauer, Arm function after stroke: from physiology to recovery, Semin. Neurol. 25 (2005) 384-395.

[20] E. Todorov, M.I. Jordan, Smoothness maximization along a predefined path accurately predicts the speed profiles of complex arm movements, J. Neurophysiol. 80 (1998) 696-714.

[21] B.R. Fajen, W.H. Warren, Behavioral dynamics of steering, obstacle avoidance, and route selection, J. Exp. Psychol. Hum. Percept. Perform. 29 (2003) 343-362.

[22] Q.-C Pham, D. Bennequin, Affine invariance of human hand movements: a direct test. ArXiv e-prints (2012) p. 1467.

[23] T. Drew, D.S. Marigold, Taking the next step: cortical contributions to the control of locomotion, Curr. Opin. Neurobiol. 33C (2015) 25-33.

[24] H. Hicheur, A.V. Terekhov, A. Berthoz, Intersegmental coordination during human locomotion: does planar covariation of elevation angles reflect central constraints? J Neurophysiol. 96 (2006) 1406-1419. 